\title{
Design and Simulation of Compound Eye Lens for Visible Light Communication and Illumination
}

\author{
Jiehui Li $(\mathbb{D}$ and Qian Zhang \\ The College of Information, Mechanical and Electrical Engineering, Shanghai Normal University (SHNU), Shanghai, China \\ Correspondence should be addressed to Qian Zhang; qianzhang@shnu.edu.cn
}

Received 28 October 2020; Accepted 1 December 2020; Published 15 December 2020

Academic Editor: Junmin Liu

Copyright ( $\odot 2020$ Jiehui Li and Qian Zhang. This is an open access article distributed under the Creative Commons Attribution License, which permits unrestricted use, distribution, and reproduction in any medium, provided the original work is properly cited.

\begin{abstract}
We proposed a scheme for designing an optical launch system that can make the light intensity more uniform on the receiving plane via a compound eye lens combined with a sunflower plano-convex lens. The simulation results demonstrate that the light converges on the optical axis after passing through the sunflower-shaped plano-convex lens array and compound eye lens. The divergence angle and central light intensity of the receiving plane are, respectively, $26.57^{\circ}$ and $80.50 \%$ of the total emitted light power for the array structure of the compact compound eye plano-convex lens, while those are $21.80^{\circ}$ and $62.50 \%$ for the discrete compound eye lens. From the above results, it can be seen that the compact compound eye lens is more conducive to the uniform distribution of light intensity on the receiving plane compared with the discrete compound eye lens, taking into account the dual application of illumination and communication.
\end{abstract}

\section{Introduction}

Visible light communication (VLC) technology is an emerging wireless optical communication technology developed on the white light emitting diode (LED) technology [1-4]. It uses LEDs to send out high-speed light and dark flashing signals that are difficult to distinguish with the naked eye to transmit information [5]. It can realize the functions of lighting and communication at the same time. Compared with traditional radio frequency communication and other wireless communication systems, visible light communication does not require radio spectrum authorization and has the advantages of good confidentiality, high transmission power, fast transmission speed, and no electromagnetic radiation and interference $[6,7]$. The key technologies of visible light communication include the selection and layout of light sources, signal modulation and demodulation, signal propagation, and reception of weak light signals [8]. For the control of the emission angle of the LED light source in the emission module of the visible light communication system, a lens is usually used.
With the gradual deepening of the research on visible light communication technology, researchers found that the communication performance of the optical receiving system in a special environment is limited [9]. For example, the background light interference of the outdoor channel environment is relatively strong, the transmission error rate of optical communication equipment in a mobile state is high, the traditional optical receiver collects less light, the field of view is small, and the superposition of the channel and conditioning circuit noise seriously affects system performance [10]. The research and development direction of the optical lens at the receiving end of the visible light communication system is small size, light weight, short focal length, high concentration efficiency, large field of view, etc. $[11,12]$. Therefore, the design and optimization of the lens are very important. A common method is to use a combination of convex lenses to condense light, but the required lens group is relatively large in actual applications, which is not conducive to integration [13]. For the high-speed, longdistance, and easy-to-use underwater wireless optical communication system that requires a relatively high degree 
of integration [14], it is necessary to design a lens group with a smaller volume and a higher light collection efficiency [15].

The compound eye is a kind of visual imaging method widely existing in nature. It has the advantages of large field of view, three-dimensional imaging, small aberrations, and miniaturization of the imaging system [16-18]. In this paper, the optimal design of the optical lens at the transmitting end of the visible light communication system is studied. The main research contents include designing a compound eye lens for the transmitting lens in the visible light communication system, changing the structure parameters of the compound eye lens, using the ray-tracing method to study its light collection efficiency and emission angle, and optimizing the design of the lens structure and parameters. Based on plano-convex microlenses, a compound eye planoconvex lens is designed. Through theoretical modeling, the lens structure parameters are optimized. The flat compound eye lens can be used as a transmitting lens in a visible light communication system, which reduces the requirements for the layout of the light source array.

\section{Design of the Sunflower-Shaped Lens Array}

For the simulation design of the sunflower-shaped 9-lamp bead LED array, it is planned to use a microlens array or a conventional lens on the sunflower-shaped 9-lamp bead LED array light board for primary light collection and then use a sunflower-shaped lens array for secondary light collection, and the lens optimizes the design to reduce the volume of the lens group while achieving high emission power and small emission angle, so as to adapt to the occasion with a higher degree of integration. Figure 1 shows the structure of the LED array and lens array. The package size of the LED lamp in the LED array is $3 \mathrm{~mm} \times 3 \mathrm{~mm}$, and the thickness of the microlens is $1.5 \mathrm{~mm}$. The diameter of the designed lens array plate is $106 \mathrm{~mm}$, and the thickness of the bottom plate is $2.5 \mathrm{~mm}$. The radius of curvature of the front surface of the central lens is $17.1 \mathrm{~mm}$, and the radius of curvature of the front surface of the outer lens is $16.6 \mathrm{~mm}$.

\section{Modeling and Simulation of Compound Eye Lens}

In the simulation system, the sunflower-shaped lens array is simulated and analyzed by TracePro. The material of the compound eye lens is polymethyl methacrylate (PMMA). The center position of the LED array light board is used as the origin of the coordinates of the XY plane. The luminous flux of each LED is set to 1 watt, the light transmission distance is $50 \mathrm{~cm}$, and the size of the detector's receiving surface is $50 \mathrm{~cm} \times 50 \mathrm{~cm}$ square plane. Figure 2 is the effect diagram obtained by ray tracing the sunflower-shaped lens array.

Figure 3 is the total-irradiance map diagram of the receiving surface of the sunflower-shaped plano-convex lens array. It can be seen from the figure that the light converges on the optical axis after passing through the sunflower- shaped plano-convex lens array, the light collection efficiency reaches $91.75 \%$, and the divergence angle is greater than $11.31^{\circ}$. It shows that the sunflower-shaped planoconvex lens array can achieve better light convergence effect.

In the visible light communication system, in order to meet the application requirements of both lighting and communication, in the design of the lens array, we need to make the light distribution uniform while ensuring that the receiving end has sufficient light intensity. In this work, we designed a compound eye lens for the visible light communication system. Figure 4 shows the array structure diagram of a compound eye plano-convex lens and the ray-tracing renderings of the sunflower-shaped lens array and compound eye plano-convex lens. The design substrate diameter is $110 \mathrm{~mm}$, the number of lenses in the compound eye lens array is 37 , and the radius of curvature is $10 \mathrm{~mm}$. The luminous flux of each LED is also set to $1 \mathrm{watt}$, the light transmission distance is $50 \mathrm{~cm}$, and the size of the detector's receiving surface is $50 \mathrm{~cm} \times 50 \mathrm{~cm}$ square plane.

Figure 5 gives the total-irradiance map diagram of the receiving surface of the compound eye plano-convex lens and sunflower-shaped plano-convex lens array. It can be seen that the light converges on the optical axis after passing through the sunflower-shaped plano-convex lens array and compound eye lens. The light collection efficiency falls to $42.35 \%$. The divergence angle is greater than $26.57^{\circ}$. The central light intensity of the receiving plane ranges from $75.00 \%$ to $80.50 \%$ of the total emitted light power for the array structure of the compact compound eye plano-convex lens. Although part of the light converged by the sunflower lens escapes the receiving range after being refracted by the compound eye lens, the light distribution at the receiving end is more uniform, which is more suitable for the application scenario where multiple discrete receivers receive simultaneously in multi-input multi-output (MIMO) visible light communication.

In order to further research the influence of the number of monocular lenses in the compound eye lens on the light collection efficiency and divergence angle, we compared the compound eye lens with 97 monocular lenses and 127 monocular lenses. Figure 6 gives the array structure diagram of a compound eye plano-convex lens with 97 monocular lenses and 127 monocular lenses. The radius of curvature is $5 \mathrm{~mm}$. The design substrate diameter is $110 \mathrm{~mm}$.

Figures 7 and 8 show the total-irradiance map diagram of the receiving surface of the compound eye plano-convex lens and sunflower-shaped plano-convex lens array when the number of monocular lenses is 97 and 127. From Figure 7, it is evident that the light collection efficiency when the number of monocular lenses is 97 is $50.51 \%$ and the divergence angle is greater than $21.80^{\circ}$. The central light intensity of the receiving plane is $62.50 \%$ of the total emitted light power for the array structure of a compact compound eye plano-convex lens. From Figure 8 , it is evident that the light collection efficiency when the number of monocular lenses is 127 is only $41.57 \%$ and the divergence angle is 


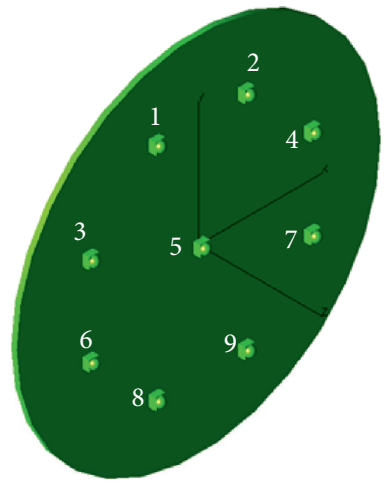

(a)

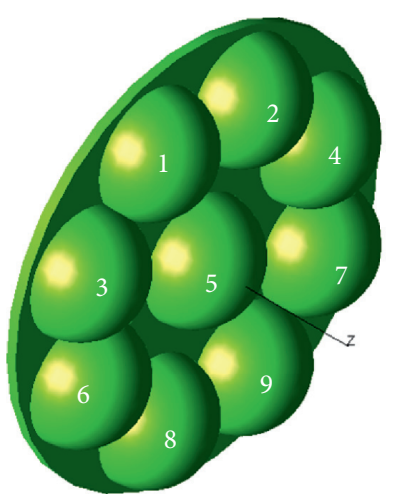

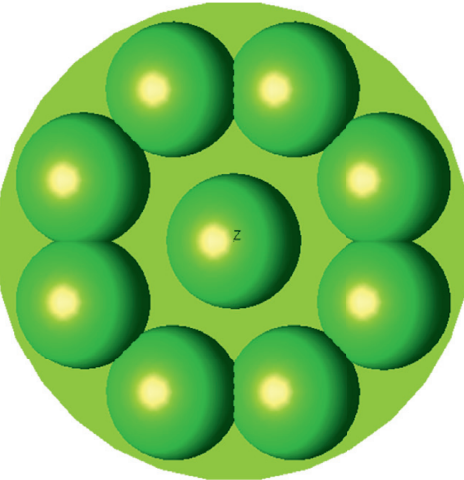

(b)

FIgURE 1: The structure of (a) LED array and (b) sunflower-shaped lens array.

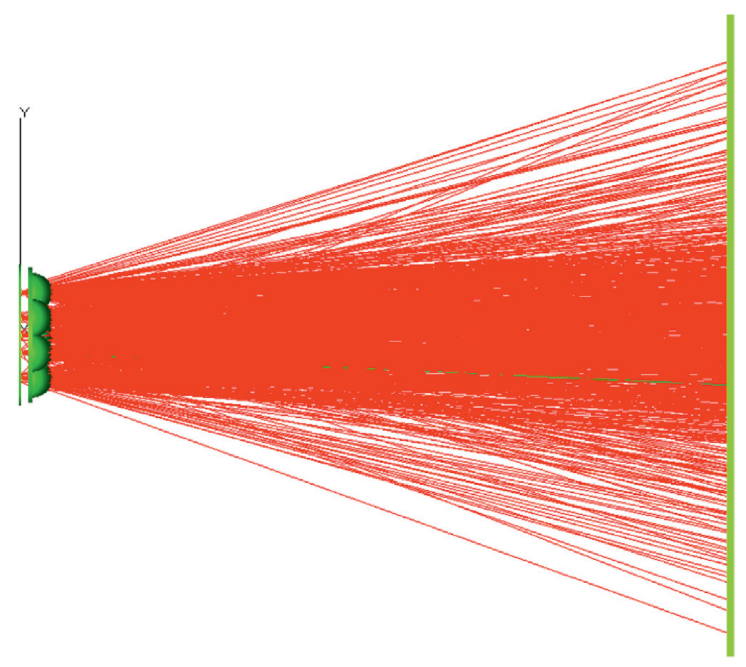

Figure 2: The effect diagram obtained by ray tracing the sunflower-shaped lens array.

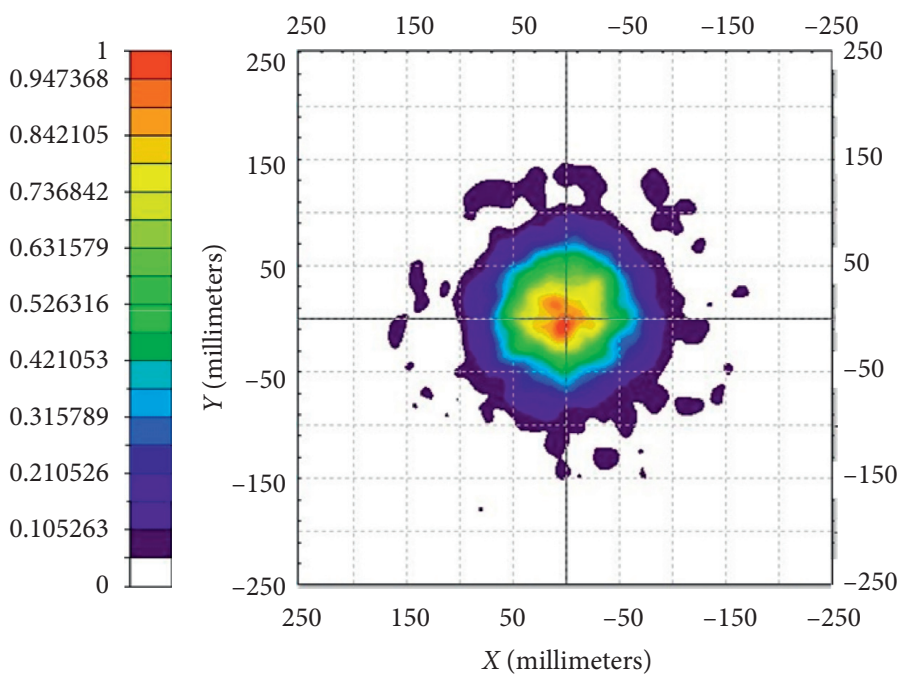

(a)

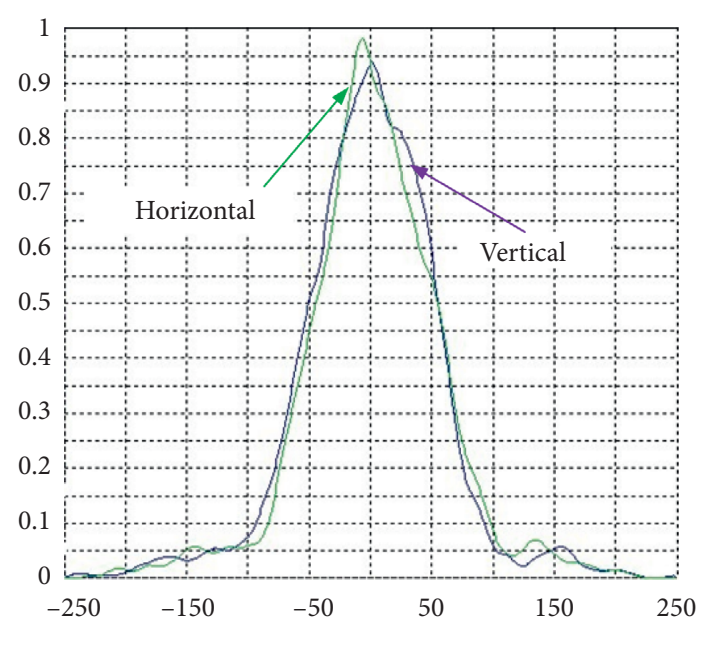

(b)

FIgURE 3: Total-irradiance map diagram of the receiving surface of the sunflower-shaped plano-convex lens array. 


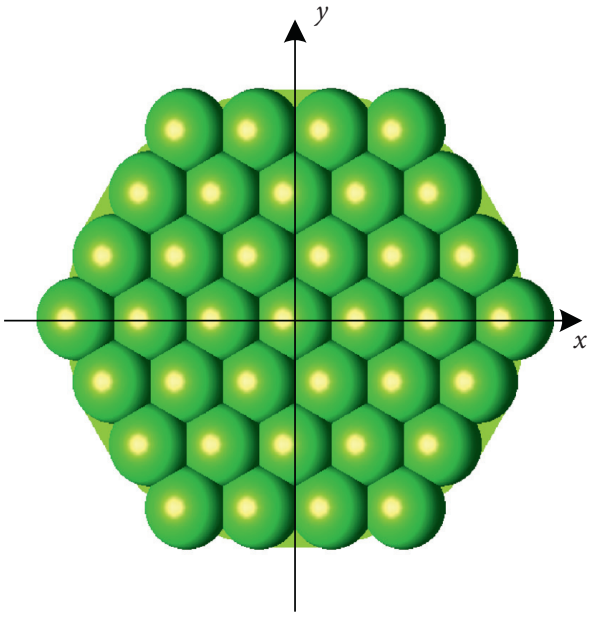

(a)

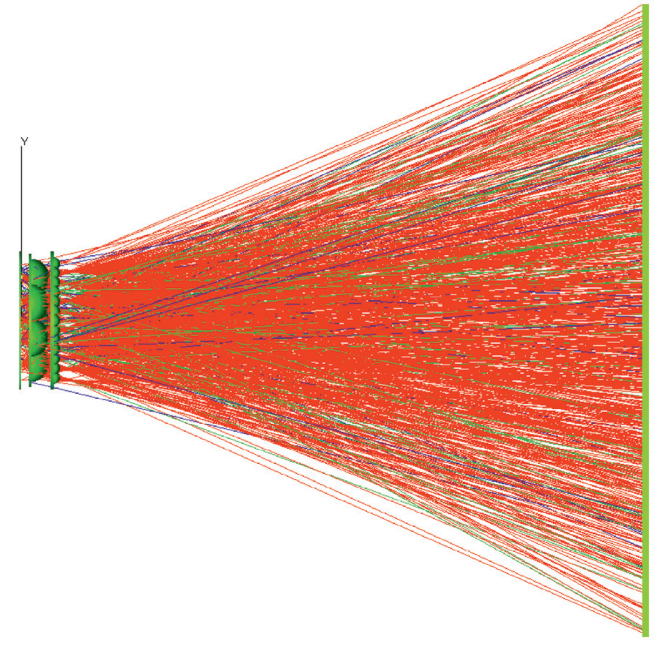

(b)

Figure 4: (a) Array structure diagram of a compound eye plano-convex lens; (b) ray-tracing renderings of the sunflower-shaped lens array and compound eye plano-convex lens.

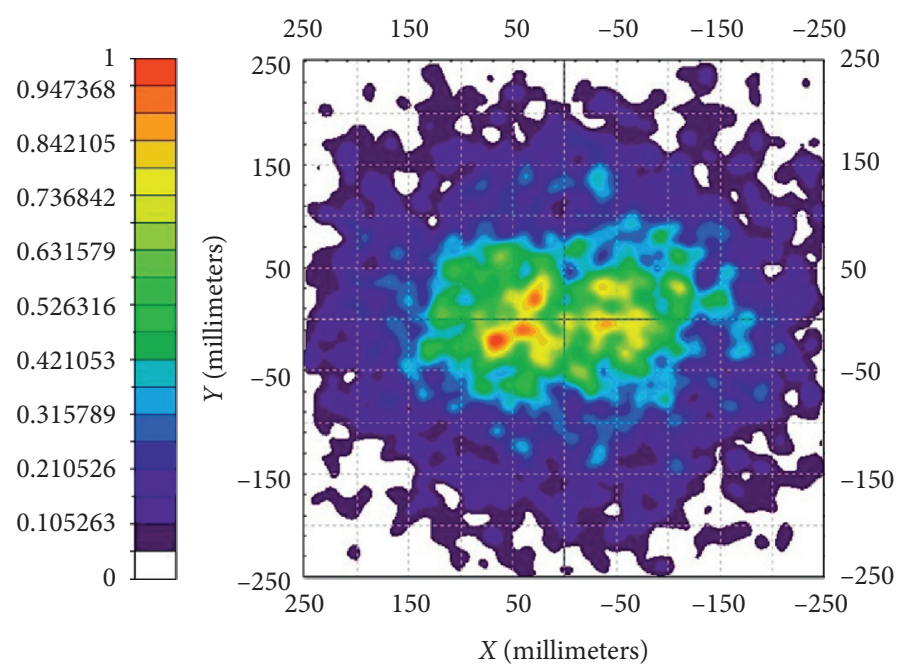

(a)

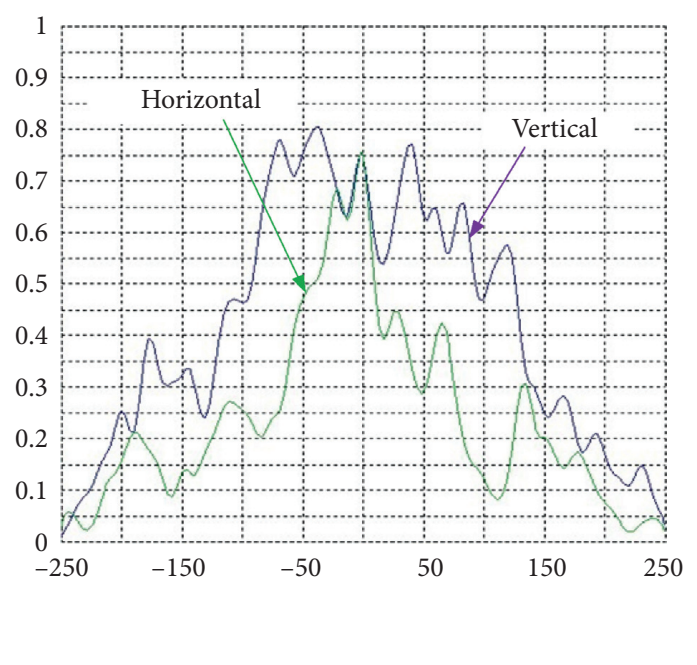

(b)

FIGURE 5: Total-irradiance map diagram of the receiving surface of the compound eye plano-convex lens with 37 monocular lenses and sunflower-shaped plano-convex lens array.

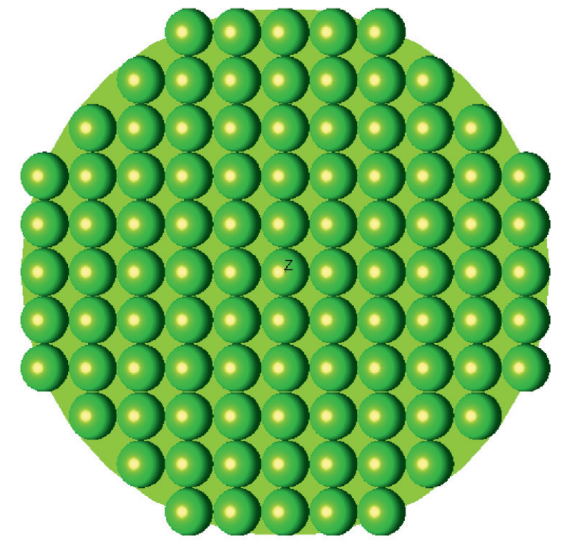

(a)

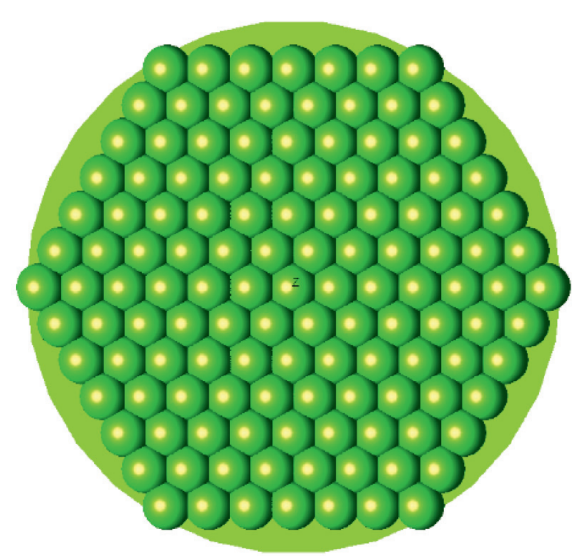

(b)

FIgURE 6: Array structure diagram of a compound eye plano-convex lens: (a) 97 monocular lenses with discrete distribution; (b) 127 monocular lenses with compact distribution. 


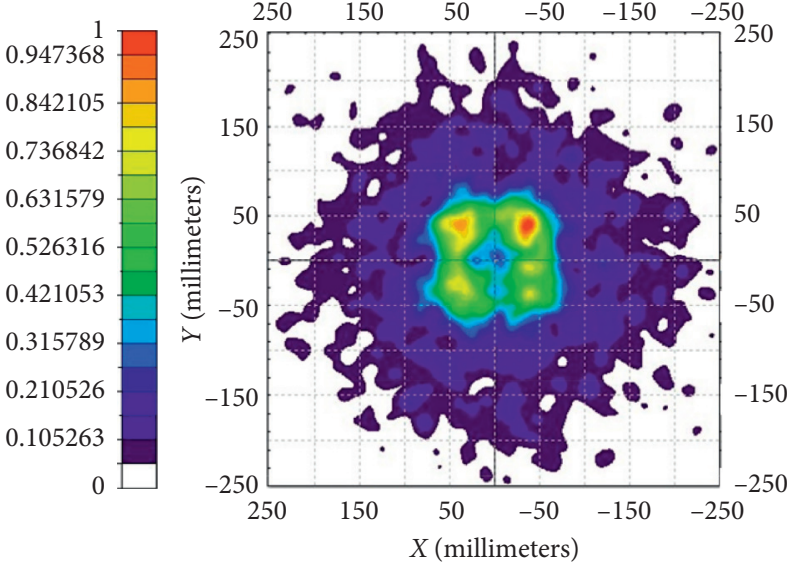

(a)

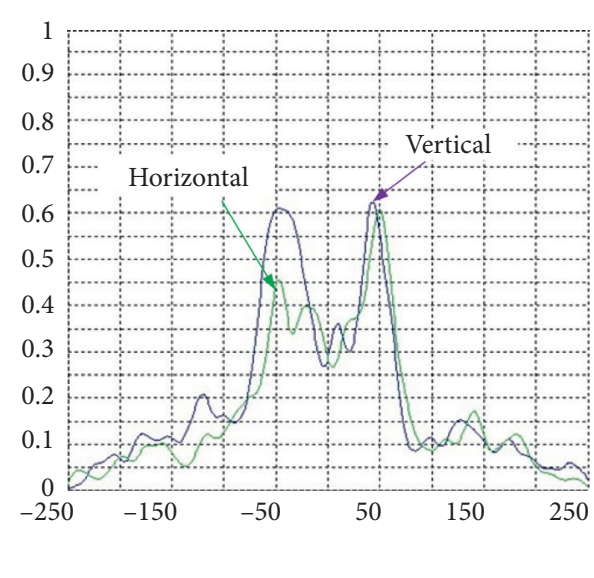

(b)

FiguRE 7: Total-irradiance map diagram of the receiving surface of the compound eye plano-convex lens with 97 monocular lenses and sunflower-shaped plano-convex lens array.

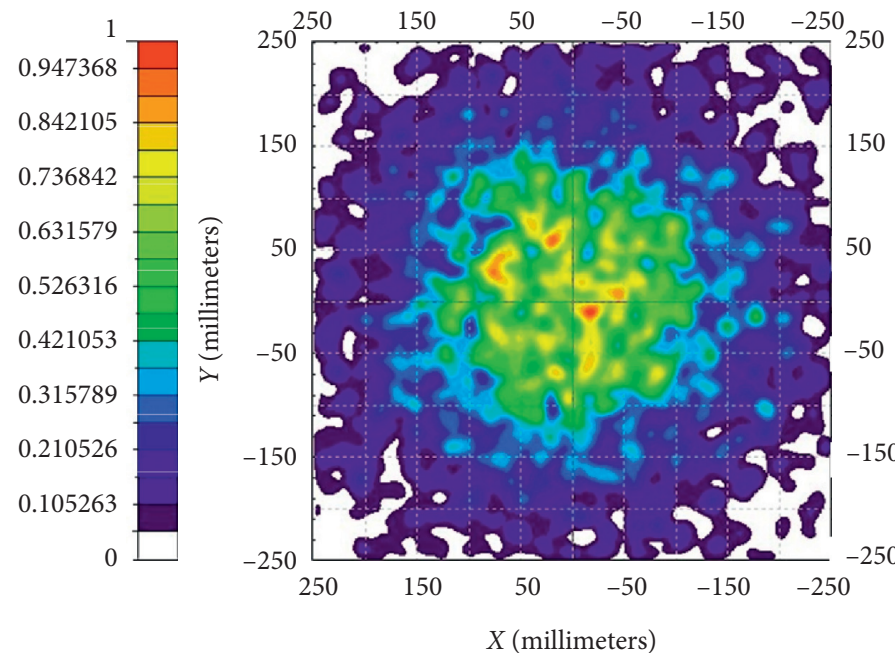

(a)

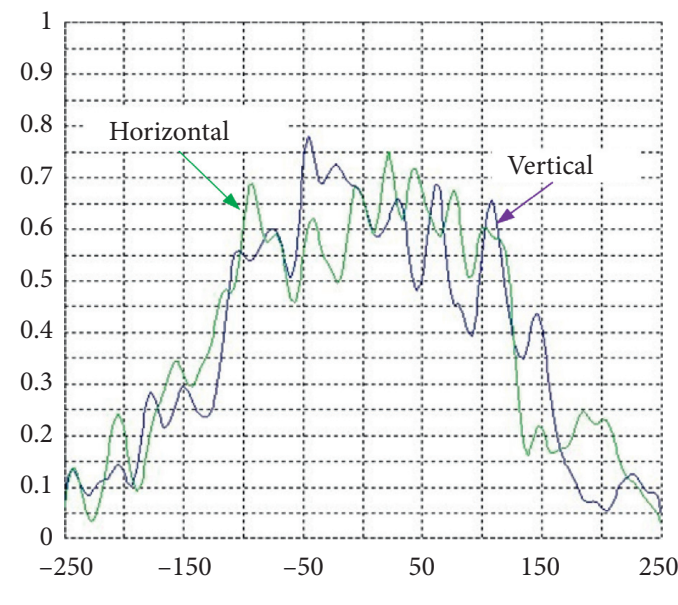

(b)

FIGURE 8: Total-irradiance map diagram of the receiving surface of the compound eye plano-convex lens with 127 monocular lenses and sunflower-shaped plano-convex lens array.

greater than $26.57^{\circ}$. From the light intensity distribution diagram of the receiving plane, it can be seen that the light is centered on the axis after the light emitted by the LED array passes through the sunflower lens and the compound eye plano-convex lens.

From Figures 5 and 8, the divergence angle and central light intensity of the receiving plane of the array structure of the compound eye lens, as shown in Figures 4(a) and 6(b), are basically the same, approximately $26.57^{\circ}$ and $75.00 \%$ to $80.50 \%$ of the total emitted light power. The light collection efficiency decreases slightly as the number of monocular lenses increases, while the light is more evenly distributed on the receiving plane. In Figure 7, the light collection efficiency of the array structure of the compound eye lens as shown in Figure 6(a) is higher than that in Figures 4(a) and 6(b), but the light intensity distribution in the receiving plane is not uniform enough. The results show that the application of the compound eye lens as the array structure of the compound eye lens as shown in Figures 4(a) and 6(b) is beneficial for taking into account lighting application and the MIMOVLC system.

\section{Conclusion}

In this paper, we designed two types of compound eye lens combined with the sunflower plano-convex lens for lighting application and MIMO-VLC systems. The simulation demonstrated the light converges on the optical axis after passing through the sunflower-shaped plano-convex lens array and compound eye lens. From the results, the compact compound eye lens is beneficial to the uniform distribution of light intensity on the receiving plane compared with the discrete compound eye lens, giving consideration to the applications of illumination and communication. 


\section{Data Availability}

No data were used to support this study.

\section{Conflicts of Interest}

The authors declare that they have no conflicts of interest.

\section{Acknowledgments}

This work was supported by the general project of Shanghai Normal University.

\section{References}

[1] P. H. Pathak, X. Feng, P. Hu, and P. Mohapatra, "Visible light communication, networking, and sensing: a survey, potential and challenges," IEEE Communications Surveys \& Tutorials, vol. 17, no. 4, pp. 2047-2077, 2015.

[2] L. E. M. Matheus, A. B. Vieira, L. F. M. Vieira, M. A. M. Vieira, and O. Gnawali, "Visible light communication: concepts, applications and challenges," IEEE Communications Surveys \& Tutorials, vol. 21, no. 4, pp. 3204-3237, 2019.

[3] T. Wu, Y. Chi, H. Wang et al., "Tricolor R/G/B laser diode based eye-safe white lighting communication beyond $8 \mathrm{Gbit} /$ s.," Scientific Reports, vol. 7, no. 1, 2017.

[4] L. Yin, W. O. Popoola, X. Wu, and H. Haas, "Performance evaluation of non-orthogonal multiple access in visible light communication," IEEE Transactions on Communications, vol. 64, no. 12, pp. 5162-5175, 2016.

[5] H. Haas, L. Yin, Y. Wang, and C. Chen, "What is LiFi?," Journal of Lightwave Technology, vol. 34, no. 6, pp. 1533-1544, 2016.

[6] A.-M. Cailean and M. Dimian, "Current challenges for visible light communications usage in vehicle applications: a survey," IEEE Communications Surveys \& Tutorials, vol. 19, no. 4, pp. 2681-2703, 2017.

[7] L. U. Khan, "Visible light communication: applications, architecture, standardization and research challenges," Digital Communications and Networks, vol. 3, no. 2, pp. 78-88, 2017.

[8] Y. Zhuang, L. Hua, L. Qi et al., "A survey of positioning systems using visible LED lights," IEEE Communications Surveys \& Tutorials, vol. 20, no. 3, pp. 1963-1988, 2018.

[9] W. Gu, M. Aminikashani, P. Deng, and M. Kavehrad, "Impact of multipath reflections on the performance of indoor visible light positioning systems," Journal of Lightwave Technology, vol. 34, no. 10, pp. 2578-2587, 2016.

[10] R. Gharat and S. S. Thorat, "A review: miller encoder for outdoor mimo vlc application," International Journal of Recent Scientific Research, vol. 8, no. 4, pp. 16303-16307, 2017.

[11] M. Z. Chowdhury, M. T. Hossan, A. Islam, and Y. M. Jang, "A comparative survey of optical wireless technologies: architectures and applications," IEEE Access, vol. 6, pp. 9819-9840, 2018.

[12] C. Wang, H.-Y. Yu, Y.-J. Zhu et al., "A long distance underwater visible light communication system with single photon avalanche diode," IEEE Photonics Journal, vol. 8, no. 5, pp. 1-11, 2016.

[13] H. Choi, J.-M. Ryu, and J.-Y. Yeom, "Development of a double-gauss lens based setup for optoacoustic applications," Sensors, vol. 17, no. 3, p. 496, 2017.

[14] J. Li, F. Wang, M. Zhao, F. Jiang, and N. Chi, "Large-coverage underwater visible light communication system based on blue LED employing equal gain combining with integrated PIN array reception," Applied Optics, vol. 58, no. 2, pp. 383-388, 2019.

[15] W. Yuan, L. Li, W. Lee et al., "Fabrication of microlens array and its application: a review," Chinese Journal of Mechanical Engineering, vol. 31, p. 16, 2018.

[16] J. J. Chen, Z. Y. Huang, T. S. Liu et al., "Freeform lens design for light-emitting diode uniform illumination by using a method of source-target luminous intensity mapping," Applied Optics, vol. 54, no. 28, pp. 146-152, 2015.

[17] B. Yu, S. Liang, Y. Yang et al., "Performance investigation on hemispherical lens used in photodetector for visible light communications," in Proceedings of the 2019 20th International Conference on Electronic Packaging Technology (ICEPT), pp. 1-4, Hong Kong, China, August 2019.

[18] Y. Wang, T. Lan, and G. Ni, "Optical receiving system based on a compound parabolic concentrator and a hemispherical lens for visible light communication," Applied Optics, vol. 55, no. 36, pp. 10229-10238, 2016. 